\section{Gangrene of the Caecum as a Complication of Foreign Body Ingestion and Closed Loop Obstruction of the Colon}

Sir,

Caecal gangrene is a rare cause of acute abdomen.1,2 It can present with right lower quadrant pain and fever or signs of peritonitis, if associated with perforation. It may be caused by occlusive or non-occlusive colonic ischaemia. Mechanical bowel obstruction causes occlusive colonic ischaemia. Closed loop obstruction occurs when two points along the course of a bowel are obstructed. This is usually caused by internal herniation, adhesions or a twist of the mesentery or volvulus, in case of colon. If closed loop obstruction is left untreated, it can lead to ischaemic changes in bowel.

Here, we report a case of a young man of 34 years with no comorbids presenting with gradually worsening abdominal pain associated with high grade fever and constipation for last three days. He gave history of eating a lot of berries with seeds five days ago. His symptoms suddenly worsened over past three hours and he had two episodes of non-bilious vomiting. On clinical examination, patient had heart rate of $140 / \mathrm{min}$ and blood pressure of $90 / 60 \mathrm{~mm}$ of $\mathrm{Hg}$ and temperature of $99^{\circ} \mathrm{F}$. He also had dehydration and tachypnea. His abdomen was tense, board-like rigid and markedly tender, suggestive of acute peritonitis. The bowel sounds were not audible. On rectal examination, seeds of berries were removed. On insertion of nasogastric tube, $250 \mathrm{ml}$ bile-stained fluid was aspirated.

A postero-anterior chest radiograph revealed free gas under diaphragm. His total leukocyte count (TLC) was $17.8 \times 109 / \mathrm{L}$ with $89 \%$ neutrophils. A diagnosis of intestinal perforation leading to peritonitis and septic shock was made. After resuscitation, patient was taken to operation theatre for exploratory laparotomy. Intraoperatively, there was mid ileal perforation and caecal gangrene, but the ileocaecal valve and appendix showed no ischemia. No vascular occlusions were found. There was faecal peritonitis with berry seeds present in peritoneal cavity.

A primary repair of ileal perforation and right hemicolectomy was done. After removing berry seeds from colon and thorough peritoneal lavage, ileostomy and mucous fistula of colon was made. Histopathologic analysis of the resected specimen showed acute inflammation showing infiltrates of neutrophils and bacteria with caecal gangrene. The stoma was reversed and ileo-transverse colon anastomosis made after 12 weeks, with an uneventful recovery.

Caecal gangrene may be caused by occlusive or nonocclusive colonic ischaemia. The occlusive forms of colonic ischemia include atherosclerosis, thromboembolization, venous occlusion, and mechanical bowel obstruction; whereas, non-occlusive includes low-flow state (shock).3,4 Mechanical bowel obstruction at two contiguous points causes closed loop obstruction. In large bowel, closed loop obstruction occurs due to volvulus or distal colonic obstruction due to malignancy, strictures, extra-luminal compression or faecal impaction with a competent ileocaecal valve. ${ }^{5}$ The valve is formed by the circular muscle fibres of the bowel and mucous membrane. 6 The superior and inferior ileocaecal ligaments also contribute to the competence. ${ }^{7}$ Manometric studies have suggested that tonic pressure at the ileocaecal valve is proportional to the bowel distention. $810-30 \%$ of patients have a competent valve on barium studies and such valves increase the chances of closed loop obstruction.

Intraluminal pressures rises greatly due to bowel distention after closed loop obstruction. The rise in intraluminal pressure leads to venous congestion, arterial occlusion and ischaemic changes. Bacterial proliferation also plays a role. If treatment is delayed, it may progress to bowel necrosis, gangrene and perforation leading to faecal peritonitis and septicaemia. 9 Such fulminant obstructive colitis leading to bowel necrosis is known as necrotising ischemic colitis.

Closed loop obstruction caused by ingestion of foreign bodies like berry seeds with a competent ileocaecal

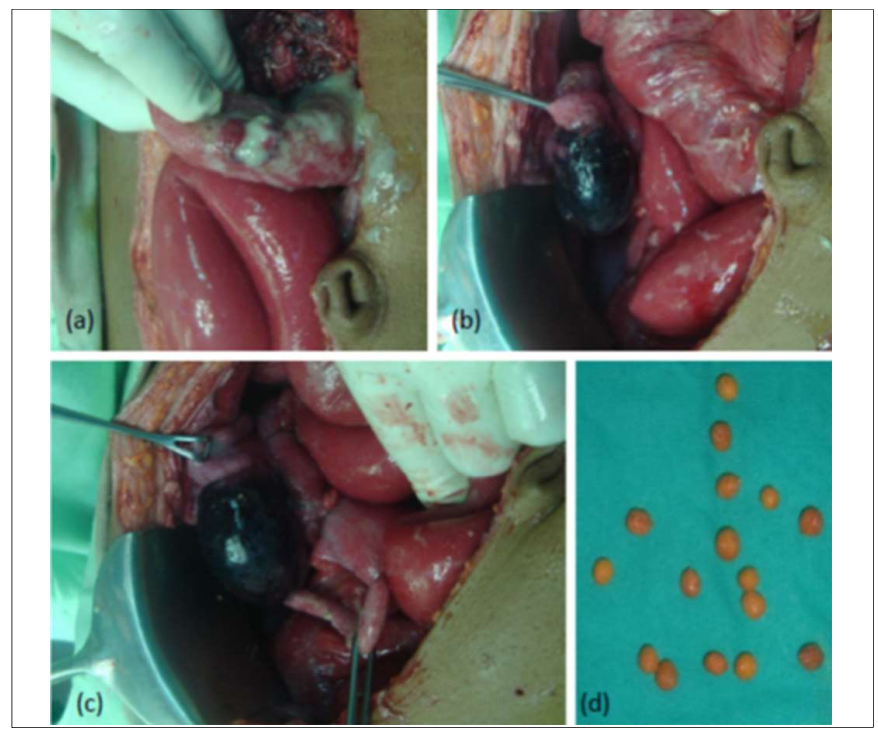

Figure 1: Intraoperative photographs showing; (a) Perforation of lleum. (b) Caecal gangrene. (c) Gangrenous caecum and healthy appendix. (d) Berry seeds. 
valve causing caecal gangrene and ileal perforation has not been described.

In the evaluation of acute abdomen, foreign body ingestion should be taken into account. A detailed history plays important role in correct diagnosis and treatment. Foreign body ingestion may lead to closed loop obstruction in the presence of competent ileocaecal valve and bowel perforation in the closed loop or in other part of bowel. The ischaemic changes may rapidly involve the obstructed colon. Therefore, prompt diagnosis and management are imperative.

\section{REFERENCES}

1. Wiesner W, Mortelé KJ, Glickman JN, Ros PR. "Cecal gangrene": a rare cause of right-sided inferior abdominal quadrant pain, fever, and leukocytosis. Emerg Radiol 2002; 9:292-5.

2. Atamanalp SS, Ozogul B, and Kisaoglu A. Cecal volvulus: A rare cause of intestinal obstruction. Eurasian J Med 2012; 44:115-6.

3. Dirican A, Unal B, Bassulu N, Tatli F, Aydin C, Kayaalp C. Isolated cecal necrosis mimicking acute appendicitis: A case series. J Med Case Rep 2009; 3:7443.

4. Bhaskaran A, Mohan A. A rare case report of colonic perforation and gangrene: a sequelae of self-inflicted transanal compressed air injury. Euroasian J Hepato-Gasteroenterol 2013; 3:136-8.

5. Weingrow D, McCague A, Shah R, Lalezarzadeh F. Delayed presentation of sigmoid volvulus in a young woman. West $J$ Emerg Med 2012; 1:100-2.

6. Bower TC. Ischemic colitis. Surg Clin North Am 1993; 73:1037-53.

7. Kumar D, Phillips SF. The contribution of external ligamentous attachments to function of the ileocaecal junction. Dis Colon Rectum 1987; 30:410-6.

8. Slam KD, Calkins S, Cason FD. La Place's law revisited: Cecal perforation as an unusual presentation of pancreatic carcinoma. World J Surg Oncol 2007; 5:14.

9. Sali PA, Pilania V, Sutar S, Krishna K, Ghetla S, Shetty T. Total colectomy in a gangrenous large bowel due to a rare double closed loop obstruction. Int J Surg Case Rep 2015; 17:1-4.

Muhammad Tahsin Hashmi ${ }^{1}$ and Amberin Taqi ${ }^{2}$

Department of Surgery 1 / Radiology ${ }^{2}$, Combined Military

Hospital, Kohat, Pakistan

Correspondence: Maj. Muhammad Tahsin Hashmi,

Department of Surgery, Combined Military Hospital, Kohat,

Pakistan

E-mail:drtahsin76@gmail.com

Received: March 16, 2018; Accepted: October 18, 2018 